\title{
Imaging in Oncology
}

\author{
M.A. Blake, M.K. Kalra, and S.T. Rosen, eds. \\ New York, NY: Springer Science + Business Media, LLC, 2008, \$129
}

The purpose of this book is to provide an overview of oncologic imaging and its application to medical and surgical therapies. Also, established use of and newer developments in the multiple-imaging modalities are discussed.

This book is aimed toward radiologists, oncologists, radiation therapists, residents, and fellows in these specialties. Other physicians managing oncology patients can also benefit from the materials presented.

The book is organized into 6 sections (20 chapters), coauthored by multiple specialists, that present an overview of various anatomic neoplasms. Section I includes chapters covering the brain, spinal neoplasms, and head and neck neoplasms, with an introductory chapter on anatomic, physiologic, and metabolic imaging. Section II is 3 chapters dedicated to neoplasms of the lungs, mediastinum, and heart. Section III is 7 chapters dealing with neoplasms of the hepatobiliary system, pancreas, colorectum, genitourinary tract, adrenal glands, peritoneum, and male reproductive tract. Section IV is 2 chapters addressing skeletal and softtissue tumors. Section V is 3 chapters discussing reticuloendothelial and pediatric malignancies and interventional radiology. Section VI is dedicated to breast tumors.
The chapters are well written and easy to understand, and the references in each chapter are comprehensive. Given that these are overview chapters, the 2 chapters that stand out are "PET Imaging of Brain Tumors" and "Extracranial Head and Neck Neoplasms: Role of Imaging."

Neoplasms of the esophagus and small bowel are not covered in this textbook, nor are any gynecologic neoplasmsa major limitation given the title of the book. Some of the images are dark and difficult to see. Each chapter has a summary of key points but at no consistent location in each chapter; some of these summaries are at the beginning and others at the end of the chapters. The conclusion at the end of chapter 5 (on thoracic malignancies) presents comments on head and neck malignancies (chapter 4).

Given its limitations as to the neoplasms that are discussed, the book does provide an overview of oncologic imaging. Other, more comprehensive, general and specialty oncologic imaging textbooks are available to interested readers.

Aurelio Matamoros, Jr. M.D. Anderson Cancer Center Houston, Texas

COPYRIGHT (c) 2009 by the Society of Nuclear Medicine, Inc.

DOI: 10.2967/jnumed.108.056242 